\title{
Complex gas kinematics in compact, rapidly assembling star-forming galaxies ${ }^{1}$
}

\author{
R. Amorín ${ }^{1}$, J.M. Vílchez ${ }^{1}$, G. F. Hägele ${ }^{2,3}$, V. Firpo ${ }^{2,3}$, E. Pérez-Montero ${ }^{1}$, and P. \\ Papaderos $^{4}$ \\ (1) Instituto de Astrofísica de Andalucía-CSIC, Glorieta de la Astronomía S/N, E-18008 Granada, Spain \\ (2) Facultad de Ciencias Astronómicas y Geofísicas. Universidad de la Plata, Paseo del Bosque S/N, 1900 \\ La Plata, Argentina \\ (3) Instituto de Astrofísica de La Plata-CONICET, Paseo del Bosque S/N, 1900 La Plata, Argentina \\ (4) Centro de Astrofísica and Faculdade de Ciências, Universidade do Porto, Rua das Estrelas, 4150-762 \\ Porto, Portugal
}

\begin{abstract}
Deep, high resolution spectroscopic observations have been obtained for six compact, strongly star-forming galaxies at redshift $z \sim 0.1-0.3$, most of them also known as green peas. Remarkably, these galaxies show complex emission-line profiles in the spectral region including $\mathrm{H} \alpha$, $[\mathrm{N}$ II $] \lambda \lambda 6548,6584 \AA$ and $[\mathrm{S} \mathrm{II}] \lambda \lambda 6717,6731 \AA$, consisting of the superposition of different kinematical components on a spatial extent of few kpc: a very broad line emission underlying more than one narrower component. For at least two of the observed galaxies some of these multiple components are resolved spatially in their 2D-spectra, whereas for another one a faint detached $\mathrm{H} \alpha$ blob lacking stellar continuum is detected at the same recessional velocity $\sim 7 \mathrm{kpc}$ away from the galaxy. The individual narrower $\mathrm{H} \alpha$ components show high intrinsic velocity dispersion $\left(\sigma \sim 30-80 \mathrm{~km} \mathrm{~s}^{-1}\right)$, suggesting together with unsharped masking HST images that star formation proceeds in an ensemble of several compact and turbulent clumps, with relative velocities of up to $\sim 500 \mathrm{~km} \mathrm{~s}^{-1}$. The broad underlying $\mathrm{H} \alpha$ components indicate in all cases large expansion velocities (full width zero intensity FWZI $\geq 1000 \mathrm{~km} \mathrm{~s}^{-1}$ ) and very high luminosities (up to $\sim 10^{42} \mathrm{erg} \mathrm{s}^{-1}$ ), probably showing the imprint of energetic outflows from SNe. These intriguing results underline the importance of green peas for studying the assembly of low-mass galaxies near and far.
\end{abstract}

Subject headings: galaxies: kinematics and dynamics — galaxies: dwarf — galaxies: evolution — galaxies: starburst

\section{INTRODUCTION}

Vigorous bursts of star-formation are key stages in the evolution of galaxies decisively influence their observational present and future integrated properties. Theoretical studies predict some bal-

\footnotetext{
${ }^{1}$ CONSOLIDER-GTC fellow;

Email: amorin@iaa.es

${ }^{1}$ Based on observations made with the William Heschel Telescope operated on the island of La Palma by the Isaac Newton Group in the Spanish Observatorio del Roque de los Muchachos of the Instituto de Astrofsica de Canarias.
}

ance between significant gas inflow and strong star formation feedback regulating the growth of galaxies, especially at increasing redshifts (e.g. Davé et al. 2012). Gas accretion, either supported by small interactions/mergers with gasrich companions or by gravity-driven motions produced by the formation and evolution of starforming clumps in dynamically young systems (e.g. Bournaud et al. 2009), can supply the metalpoor gas to feed the current starburst on galactic scales.

On the other hand, the removal of enriched gas 
by $\mathrm{SNe}$ and stellar winds in low-mass starburst galaxies, promotes substantial chemical evolution (e.g. Tenorio-Tagle et al, 2003; Recchi \& Hensler 2007), favors the cessation of the current starburst episode (e.g. Oppenheimer \& Davê 2006), and under some conditions, could lead to positive feedback (e.g. Tenorio-Tagle et al. 2005). From the observational point of view, tackling the above issues is extraordinary challenging and requires high quality observations.

Studying low-mass starburst galaxies in the local Universe can provide key insights on the mechanisms giving origin and regulating enhanced star formation activity, under physical conditions approaching those in star-forming galaxies at higher redshifts. This is the case of a rare subset of lowmass galaxies at redshift $z \sim 0.1-0.3$, also referred to as green peas (GP) Cardamone et al, 2009). These extreme emission-line galaxies are rapidly growing systems characterized by their compactness, low metallicity and unusually high specific star formation rates (sSFR $10^{-7}-10^{-9} \mathrm{yr}$ ), well in the range of those of high-redshift galaxies (e.g. Bauer et al. 2005).

In many aspects the GPs are identifiable with extreme versions of nearby blue compact dwarfs (BCD) galaxies, probably representing a major episode in their assembly history. This conclusion relies on recent results from detailed studies on their physical properties and chemical abundances, integrated star formation histories $(\mathrm{SFH})$, and photometric structure Amorín et al. 2010, 2012). They showed that GPs are currently producing a significant fraction (up to 20\%) of their total stellar mass $\left(M_{\star} \sim 10^{8}-10^{10} M_{\odot}\right)$ in a galaxywide starburst that takes place over a small $(\sim 2-$ $3 \mathrm{kpc}$ ) low-surface brightness exponential envelope, which might be due to more evolved stars. Extended nebular emission excited by a strong ongoing starburst can, however, also produce a large exponential envelope, mimicking a stellar disk (Papaderos \& Östlin 2012). Interestingly, the ionized gas-phase in these galaxies show low oxygen and high nitrogen-to-oxygen ratios, clearly deviating from the median for local galaxies of the same stellar mass.

All these properties led Amorín et al. 2010) to suggest hydrodynamical effects e.g., massive inflows and/or enriched outflows, as playing a key role before and during the short and extreme phase of mass growth where these dwarfs are seen as GPs.

In order to further explore this hypothesis, we are conducting a comprehensive study of the ionized gas kinematics and chemodynamics in these low-mass starbursts. In this letter we present first outstanding results on the remarkably complex kinematics of a handful of GPs observed using very deep, high resolution long-slit spectroscopy.

\section{SAMPLE OF GALAXIES}

The complete designations of the Sloan Digital Sky Survey (SDSS) for the observed sample of galaxies are included in Table 田. The sample consists of five galaxies at $z \sim 0.2-0.3$ from Cardamone et al. 2009), and one nearby galaxy at $z=0.1$ selected from a larger sample of strong emission-line galaxies (Amorin et al., in prep.) and included in Pilvugin et al. 2012). All the galaxies are very compact $\left(r_{50} \leq 1 \mathrm{kpc}\right)$, luminous $\left(M_{\mathrm{B}} \sim-20\right)$, and metal-poor $\left(Z / Z_{\odot} \sim 1 / 5\right)$, rapidly star-forming systems $\left(\mathrm{sSFR}=\mathrm{SFR} / \mathrm{M}_{\star} \geq 10^{-9} \mathrm{yr}^{-1}\right.$ ) with no spectral signs of non-thermal ionization due to AGNs Cardamone et al. 2009).

\section{OBSERVATIONS}

High-resolution spectroscopy was obtained in July 2011 as part of a longer-term project using the Intermediate Dispersion Spectrograph and Imaging System (ISIS) on the 4.2-m William Herschel Telescope (WHT) of the Isaac Newton Group (ING) at the Roque de los Muchachos Observatory (La Palma, Spain). We used the TEK4 CCD attached to the red arm. The R1200R grating was used in three different set-ups, selecting spectral ranges and central wavelengths around the $\mathrm{H} \alpha$ emission line, depending on the redshift of the target. For this configuration the spatial resolution of the observations was 0.44 arcsec pixel ${ }^{-1}$, and the spectral dispersion and FWHM effective resolution measured on the sky lines were $0.24 \AA$ pixel $^{-1}$ and $0.52 \AA$, respectively. The spectra were taken in several exposures along the parallactic angle, with a slit width of 0.9 arcsec. Seeing conditions varied between 0.7 and 1.5 arcsec during the run. Total exposure times were about 2 hours per galaxy.

\footnotetext{
${ }^{2}$ Through this paper we assumed a standard cosmology with $H_{0}=70, \Omega_{\Lambda}=0.7$, and $\Omega_{m}=0.3$
} 
The data was fully reduced with usual procedures (bias, overscan, flat-fielding, co-addition and cosmic ray removal) using IRAH3. Wavelength calibration was done using $\mathrm{CuNe}+\mathrm{CuAr}$ lamp arcs with an accuracy of about $0.02 \AA$. Full details of the instrumental set-up, data reduction and calibrations will be published in Amorin et al. (in prep).

\section{RESULTS}

\subsection{D-spectra}

Multiple kinematical components for each emission line can be identified from the 2D-spectra in all the observed galaxies. In Figure 1 we show examples of the long-slit spectrum in different wavelength ranges including $\mathrm{H} \alpha$. In the case of J1615 we also included the $[\mathrm{O} \mathrm{I}] \lambda 6300 \AA$ and [S II] $\lambda \lambda 6717,6731 \AA$, while for J1454 we only included the second one since the $[\mathrm{O} \mathrm{I}] \lambda 6300 \AA$ line is out of the observed spectral range.

For J1615 all the lines detected in the spectrum, even those of lower S/N (e.g., [O I] $\lambda 6300 \AA$ or [N II] $\lambda \lambda 6548,6584 \AA$ ), show double-peaked emission. This feature extents to the whole optical range, as noticed by Pilyugin et al. 2012) using SDSS data. Intriguing enough, the secondary narrow component is offset in the spatial direction from the main component and the stellar continuum. In addition, $\mathrm{H} \alpha$ in this galaxy shows very broad wings and a fainter component, that appears as a "bridge" between the two main narrow components.

Though with substantially lower S/N, for J1454 we found a similar situation to that seen in J1615. Two narrow components are distinguishable, at least for $\mathrm{H} \alpha$. The secondary component also appears spatially resolved. For the $[\mathrm{N} \mathrm{II}]$ and $[\mathrm{S} \mathrm{II}]$ doublets a hint of this complex spatial structure is visible as well.

A secondary $\mathrm{H} \alpha$ narrow component is also spatially resolved in J1439. Because of its low surface brightness it is barely seen above the broad component. On the other hand, an adjacent purely $\mathrm{H} \alpha$ emitter is projected $\sim 14$ arcsec to the SW in the slit with the same radial velocity of J1439.

\footnotetext{
${ }^{3}$ IRAF: the Image Reduction and Analysis Facility is distributed by NOAO, operated by AURA, Inc., under agreement with the NSF
}

This companion at $\sim 7 \mathrm{kpc}$ exhibits only pure $\mathrm{H} \alpha$ emission in the spectrum, and no counterpart is detected in SDSS imaging.

\subsection{Analysis of emission line profiles}

Since it was not possible to separate the different and relatively close spatial components in their corresponding 1D-spectra, the integrated 1D-spectra in the region of $\mathrm{H} \alpha$ and $[\mathrm{N} \mathrm{II}] \lambda \lambda 6548,6584 \AA$ was used to analyze the structure of the emission line profiles. This allowed us to verify the presence of multiple components and to characterize their kinematics. In doing so, we adopted the technique presented by Hägele et al. 2007) and modified later by Firpo et al. (2010). This technique was also successfully applied in other previous studies (e.g., Hägele et al. 2011, 2012; Firpo et al. 2011). As described in detail in Firpo et al. (2010), the method involves an iterative fitting of multiple Gaussian components using the task NGAUSSFIT in IRAF. Starting from the simplest solution, where the line profiles are formed by a broad- and a narrow-line component, the procedure adds extra Gaussian components until the solution that best fits the observed profile is obtained. Using this technique we obtain the different Gaussian parameters for each component, allowing us to derive the radial velocity and the intrinsic velocity dispersions, the latter were corrected from instrumental and thermal broadening. We considered here an instrumental width $\sigma_{i}=10.2 \mathrm{~km} \mathrm{~s}^{-1}$, as measured from the observed lamp arcs. The thermal contribution was estimated assuming a kinetic temperature of $T=1.2 \times 10^{4} \mathrm{~K}$ Amorín et al. 2010, 2012. Fluxes (and corresponding uncertainties) for each component were derived from the amplitude and the FWHM of the Gaussian component.

Figures 2 3 show the results of the NGAUSSFIT fitting procedure overimposed to the observed $\mathrm{H} \alpha$ and $[\mathrm{N} \mathrm{II}] \lambda \lambda 6548,6584 \AA$ emission lines for the sample. All the galaxies show complex line profiles, as shown by their fit solution. The emission lines are well described with an underlying broad component and more than one narrower component. The kinematical centers of low-luminosity narrow and broad components are, in most cases, offset from those of the main narrow lines, producing the net effect of a clear asymmetry in the global line profiles. In general, and in spite of the 
their relatively low $\mathrm{S} / \mathrm{N}$, the solutions found for the $\mathrm{H} \alpha$ profiles have provided a good initial guess for the fitting of the [N II] doublet, whose final fits are in very good agreement with the $\mathrm{H} \alpha$ ones. An exception is J0040, where [N II] lines are too faint for a fitting attempt. The accuracy of the fit in the wings of the $\mathrm{H} \alpha$ emission is highlighted in the inset of Figures 23

Results from the $\mathrm{H} \alpha$ fitting, including velocity dispersions and the fractional emission measures (in \%) for each kinematical component are presented in Table 1

All the six observed galaxies show a composite of more than one strong narrow $\mathrm{H} \alpha$ components, spanning in a large range of velocity dispersions $\left(\sigma \sim 10-120 \mathrm{~km} \mathrm{~s}^{-1}\right)$ and luminosities $\left(\mathrm{L}_{H \alpha} \sim 10^{40}-10^{42} \mathrm{erg} \mathrm{s}^{-1}\right)$. With the exception of $\mathrm{J} 2325$, the $\mathrm{H} \alpha$ narrow components are blueor red-shifted with respect to the line centroid by about $\Delta \mathrm{v} \sim 50-500 \mathrm{~km} \mathrm{~s}^{-1}$. Especially remarkable are the cases of J1439, J1615, and J1454, which presents shifts between some of their components larger than $350 \mathrm{~km} \mathrm{~s}^{-1}$. In two of these galaxies at least one secondary narrow component is spatially identified in their spectra, being located on a spatial extent $\lesssim 5-10 \mathrm{kpc}$.

For the broad emission we found velocity dispersions and full width at zero intensity (FWZI) in the range of $\sigma \sim 100-250 \mathrm{~km} \mathrm{~s}^{-1}$ and $\sim 650-1750$ $\mathrm{km} \mathrm{s}^{-1}$, respectively. The corresponding broad $\mathrm{H} \alpha$ luminosities are exceedingly large $\sim 5 \times 10^{41}$ $1.5 \times 10^{42} \mathrm{erg} \mathrm{s}^{-1}$, representing $\sim 40-65 \%$ of the total $\mathrm{H} \alpha$ emission (Table 1). Only J1615 shows the broad emission significantly red-shifted $(\sim 100$ $\mathrm{km} \mathrm{s}^{-1}$ ) from the line centroid. Remarkably, forbidden lines, such as [N II] also show broad components.

\section{DISCUSSION}

\subsection{The broad component suggest rapid gas flows}

The broad emission in the wings of emission lines suggests very high velocity gas. Different mechanisms have been explored in the literature to account for it in both giant extragalactic HII regions (GEHRs) (e.g. Diaz et al. 1987: Castañeda et al. 1990) and BCDs (e.g. Izotov et al. 2007; James et al. 2009). These typically include (1) strong stellar winds caused by hot, massive stars, e.g., WR, Ofp, and LBV stars, (2) expansion of multiple SNe remnants, (3) SNedriven superbubble blow-up, (4) effects of turbulent mixing layers (TML), and (5) AGNs.

The presence of large amounts of WR stars has been confirmed for two galaxies of the sample - J0040 and J2325 - using high S/N OSIRISGTC spectroscopy Amorín et al. 2012. For some other GPs (e.g. J1615 and J1439) WR features are already detectable from SDSS spectra Hawley 2012). Besides that, the strong ongoing starburst activity in GPs Amorín et al. 2012 and their clear detection in the radio continuum Chakraborti et al. 2012) are consistent with a significant number of WR stars and SNe. Both dense circumstellar envelopes of hot massive stars with strong stellar winds (e.g., WRs) and SNe remnants can produce broad components with luminosities of about $10^{36}-10^{39} \mathrm{erg} \mathrm{s}^{-1}$ and expansion velocities of $>1000 \mathrm{~km} \mathrm{~s}^{-1}$ Izotov et al. 2007). Our calculations confirm that the mechanical energy released by the SNe II expected from the measured broad $\mathrm{L}(\mathrm{H} \alpha)$ using Starburst 99 models Leitherer et al. 1999) at the appropriate $Z$ is fully consistent with their measured dispersions. Therefore, the combined effects of (1) and (2) appear as the probable dominant source for the observed broad emission.

Other mechanisms like (3) and (4) appear unlikely as the sole explanation for the broad emission at kiloparsec scales. For example, the expansion velocity of a SNe-driven superbubble in blow-up phase is generally higher by a factor of $\sim 2-3$. If present, TMLs do not appear to be a dominant effect at global scales. Moreover, models show TMLs only producing broad emission in Balmer lines but not in forbidden lines Binette et al. 2009), as observed in our galaxies. These broad components in the forbidden lines were also observed in circumnuclear regions (Hägele et al. 2007, 2009, 2010), GEHRs Firpo et al 2010), and star-forming knots of the BCD Haro 15 (Firpo et al. 2011).

Very broad line emission with luminosities between $10^{40}-10^{42} \mathrm{erg} \mathrm{s}^{-1}$ are also expected in galaxies with AGN as due to accretion onto an intermediate-mass black hole. The existence of rare low-metallicity dwarf galaxies with AGN have been proposed in the literature (e.g. Izotov et al. 2007, 2009). Although we cannot rule it out, at 
this point the data for the GPs studied as yet do not support this hypothesis. Emission line ratios are consistent with pure starbursts and there is no evidence of hard non-thermal emission in none of these galaxies. A deeper examination of spectra looking for additional clues evidencing nuclear activity, e.g. presence of high ionization ions, and IFU data for studying the spatial distribution of the broad emission in these galaxies, are strongly required to reach more firm conclusions about this hypothesis.

Overall, the kinematics of the ISM on kpc scales in the studied galaxies is likely witnessing strong star formation feedback. So we conclude that broad emission in both Balmer and forbidden lines are mostly originated in strong gas outflows driven by the intense, galaxy-wide starburst taking place in these galaxies.

\subsection{Multiple kinematical components from emission-line fitting suggest multiple star-forming clumps}

Decomposition of emission lines in multiple kinematical components with large velocity dispersions and luminosities suggest that the starburst episode takes place in several massive clumps. Ensembles of star-forming knots/clumps distributed across the host galaxy are typical for nearby BCDs (e.g. Cairós et al. 2001) and in compact starbursts at higher redshifts (e.g. Elmegreen et al. 2005). To gain further insight into the properties of the star-forming components in GPs, we processed archival HST/ACS image 4 of J2325 and J0040 in the filter F150LP with a flux-conserving unsharp-masking technique Papaderos et al. 1998). The unsharp-masked images of these two GPs (Figure 4) reveal a wealth of morphological substructure, notably three and six knots in J2325 and J0040, respectively, with a projected separation between $\sim 0.4$ and $\sim 1 \mathrm{kpc}$. Interestingly, the number of photometrically detected knots is in both GPs equal or larger than the kinematically distinct components revealed from NGAUSSFIT. The compactness of GPs precludes a morphological analysis based on groundbased imaging from the SDSS. Despite this, for the nearest galaxy (J1615), the SDSS gri image after unsharp-masking reveails two main knots

$\overline{{ }^{4} \mathrm{HST} \text { proposal ID } 11107}$ along the slit (a and $\mathrm{c}$ in Figure 4), that can be associated with the two main narrow components in the spectrum.

Most BCD/Hit galaxies show emission lines accurately fitted using single narrow Gaussians and, eventually, one relatively broad component (e.g. Bordalo \& Telles 2011). The velocity dispersion of the main narrow component in our sample galaxies is considerably higher than the average measured turbulent velocity dispersions of GEHRs (e.g., Firpo et al. 2005, 2010), and still higher than those in local BCD/HII galaxies of similar broad-band luminosity (e.g., Terlevich \& Melnick 1981: Guzmán et al. 1996; Östlin et al. 2001; Marquart et al. 2007). Together with their compactness, these values are more consistent with those of strongly star-forming galaxies of similar luminosity at higher redshifts (e.g. Koo et al. 1995: Wisnioski et al. 2012), rather than for nearby BCDs.

We interpret the composite profiles as an evidence of multiple massive star-forming clumps, distributed in a very small (few $\mathrm{kpc}$ ) and dynamically young host galaxy. In line with results from both optical Green et al, 2010) and near infrared integral field spectroscopy (IFS) Goncalves et al. 2010) for UV-luminous starburst galaxies at $z \sim 0.1-0.3$, the observed high velocity dispersion suggests disturbed kinematics, likely driven by turbulence rather than rotation. Qualitatively, gravity (e.g. Terlevich \& Melnick 1981), shocks (e.g. Tenorio-Tagle et al. 1997), accretion (e.g. Elmegreen \& Burkert 2010), and star formation feedback (e.g. Lehnert et al. 2009) appear as possible mechanisms to inject energy to drive such high velocity dispersion.

\subsection{Clues for the triggering and regula- tion of star formation in "pea" galax- ies}

For half of the galaxies - J1615, J1439 and $\mathrm{J} 1454$, - the large difference in radial velocity found between different components (clumps) can possibly be interpreted as a sign of (clump-clump) interaction or minor-mergers. Mergers and tidal interactions with gas-rich, low-mass companions have been suggested as the main triggering mechanism for starbursts in nearby luminous BCDs (e.g., Ostlin et al, 2001; Bergvall \& Ostlin 2002). These systems, closely resembling high-redshift Lyman 
break galaxies Overzier et al, 2008), offer us a laboratory to study at high spatial resolution collisionally induced star formation and its rôle on galaxy buildup.

In the remaining galaxies, where signs of interactions are not obvious, gravitational instabilities may be the main cause for the enhanced star formation. Models suggest that large gaseous clumps formed by gravitational instabilities in primordial disks can drive significant and fast gas accretion, and trigger and sustain starburst episodes Bournaud et al. 2007). Those clumps massive enough $\left(\gtrsim 10^{7} \mathrm{M}_{\odot}\right)$ to resist disruption by star-formation can eventually coalesce toward the center, loss angular momentum, and form a spheroidal system (e.g., a bulge) in $\lesssim 1$ Gyr Noguchi 1999). This mechanism, has been proposed for bursty systems at $z \gtrsim 1$ Elmegreen et al. 2009), and suggested for some clumpy BCDs in the local Universe Elmegreen et al. 2012).

In summary, observations for the six starburst galaxies presented here suggest that these systems are likely clumpy and highly turbulent, and with strong gas flows, probably as a consequence of strong star formation feedback.

The above results highlight the important analogies found between the some local low-mass starbursts, like the GPs, and star-forming galaxies at high redshift. A further study with additional observations and including an analysis of physical properties and chemical abundances for the different kinematical component will be presented in a forthcoming paper. Furthermore, high - spectral and spatial - resolution studies using IFS, tracing both star formation and gas kinematics, appear essential to test models and disentangle the striking gas kinematics of the GPs.

We thank the referee for his/her thoughtful comments. RA gratefully acknowledges the hospitality of the Grupo de Estrellas Massivas at the IALP/FCAGLP, where part of this work was carried out. We thank G. Bosch and A. MonrealIbero for helpful suggestions on the data analysis. We also thank C. Muñoz Tuñón, G. Tenorio-Tagle, and R. Terlevich for useful comments. This work has been funded by grants, AYA2010-21887-C0401: Estallidos de Formación Estelar en Galaxias http://estallidos.iac.es/estallidos/, and CSD2006-00070: First Science with the GTC http://wWw.iac.es/consolider-ingenio-gtc/ of the Consolider-Ingenio 2010 Program, by the Spanish MICINN. P. Papaderos is supported by a Ciencia 2008 contract, funded by FCT/MCTES (Portugal) and POPH/FSE (EC). Facilities:WHT (ISIS), HST (ACS), SDSS.

\section{REFERENCES}

Amorín, R. O., Pérez-Montero, E., \& Vílchez, J. M. 2010, ApJ, 715, L128

Amorín, R., Pérez-Montero, E., Vílchez, J. M., \& Papaderos, P. 2012, ApJ, 749, 185

Bauer, A. E., Drory, N., Hill, G. J., \& Feulner, G. 2005, ApJ, 621, L89

Bergvall, N., Östlin, G. 2002, A\&A, 390, 891

Binette, L., Flores-Fajardo, N., Raga, A. C., Drissen, L., \& Morisset, C. 2009, ApJ, 695, 552

Bordalo, V., \& Telles, E. 2011, ApJ, 735, 52

Bournaud, F., Elmegreen, B. G., \& Elmegreen, D. M. 2007, ApJ, 670, 237

Bournaud, F., Elmegreen, B. G., \& Martig, M. 2009, ApJ, 707, L1

Cairós, L. M., Caon, N., Vílchez, J. M., GonzálezPérez, J. N., \& Muñoz-Tuñón, C. 2001, ApJS, 136,393

Cardamone, C., et al. 2009, MNRAS, 399, 1191

Castañeda, H. O., Vilchez, J. M., \& Copetti, M. V. F. 1990, ApJ, 365, 164

Chakraborti, S., Yadav, N., Cardamone, C., \& Ray, A. 2012, ApJ, 746, L6

Davé, R., Finlator, K., \& Oppenheimer, B. D. 2012, MNRAS, 421, 98

Diaz, A. I., Terlevich, E., Pagel, B. E. J., Vilchez, J. M., \& Edmunds, M. G. 1987, MNRAS, 226, 19

Elmegreen, D. M., Elmegreen, B. G., Rubin, D. S., \& Schaffer, M. A. 2005, ApJ, 631, 85

Elmegreen, B. G., Elmegreen, D. M., Fernandez, M. X., \& Lemonias, J. J. 2009, ApJ, 692, 12 
Elmegreen, B. G., \& Burkert, A. 2010, ApJ, 712, 294

Elmegreen, B. G., Zhang, H., \& Hunter, D. 2012, arXiv: 1201.3658

Firpo, V., Bosch, G., \& Morrell, N. 2005, MNRAS, 356,1357

Firpo, V., Bosch, G., Hägele, G. F., \& Morrell, N. 2010, MNRAS, 406, 1094

Firpo, V., Bosch, G., Hägele, G. F., Díaz, Á. I., \& Morrell, N. 2011, MNRAS, 414, 3288

Green, A. W., Glazebrook, K., McGregor, P. J., et al. 2010, Nature, 467, 684

Gonçalves, T. S., et al. 2010, ApJ, 724, 1373

Guzmán, R., Koo, D. C., Faber, S. M., et al. 1996, ApJ, 460, L5

Hägele, G. F., Díaz, Á. I., Cardaci, M. V., Terlevich, E., \& Terlevich, R. 2007, MNRAS, 378, 163

Hägele, G. F., Díaz, Á. I., Cardaci, M. V., Terlevich, E., \& Terlevich, R. 2009, MNRAS, 396, 2295

Hägele, G. F., Díaz, Á. I., Cardaci, M. V., Terlevich, E., \& Terlevich, R. 2010, MNRAS, 402, 1005

Hägele, G. F., García-Benito, R., Pérez-Montero, E., et al. 2011, MNRAS, 414, 272

Hägele, G. F., Firpo, V., Bosch, G., Díaz, Á. I., \& Morrell, N. 2012, MNRAS, 2798

Hawley, S. A. 2012, PASP, 124, 21

Izotov, Y. I., Thuan, T. X., \& Guseva, N. G. 2007, ApJ, 671, 1297

Izotov, Y. I., Guseva, N. G., Fricke, K. J., \& Papaderos, P. 2009, A\&A, 503, 61

James, B. L., Tsamis, Y. G., Barlow, M. J., et al. 2009, MNRAS, 398, 2

Koo, D. C., Guzman, R., Faber, S. M., et al. 1995, ApJ, 440, L49

Lehnert, M. D., Nesvadba, N. P. H., Le Tiran, L., et al. 2009, ApJ, 699, 1660
Leitherer, C., Schaerer, D., Goldader, J. D., et al. 1999, ApJS, 123, 3

Marquart, T., Fathi, K., Östlin, G., et al. 2007, A\&A, 474, L9

Noguchi, M. 1999, ApJ, 514, 77

Oppenheimer, B. D., \& Davé, R. 2006, MNRAS, 373,1265

Östlin, G., Amram, P., Bergvall, N., et al. 2001, A\&A, 374, 800

Overzier, R.A., Heckman, T.M., Kauffmann, G., et al. 2008, ApJ, 677, 37

Papaderos, P., Izotov, Y. I., Fricke, K. J., Thuan, T. X., \& Guseva, N. G. 1998, A\&A, 338, 43

Papaderos, P., \& Östlin, G. 2012, A\&A, 537, A126

Pilyugin, L. S., Zinchenko, I. A., Cedrés, B., et al. 2012, MNRAS, 419, 490

Recchi, S., \& Hensler, G. 2007, A\&A, 476, 841

Terlevich, R., \& Melnick, J. 1981, MNRAS, 195, 839

Tenorio-Tagle, G., Munoz-Tunon, C., Perez, E., \& Melnick, J. 1997, ApJ, 490, L179

Tenorio-Tagle, G., Silich, S., \& Muñoz-Tuñón, C. 2003, ApJ, 597, 279

Tenorio-Tagle, G., Silich, S., Rodríguez-González, A., \& Muñoz-Tuñón, C. 2005, ApJ, 628, L13

Wisnioski, E., Glazebrook, K., Blake, C., et al. 2012, arXiv:1203.0309

This 2-column preprint was prepared with the AAS IATEX macros v5.2. 

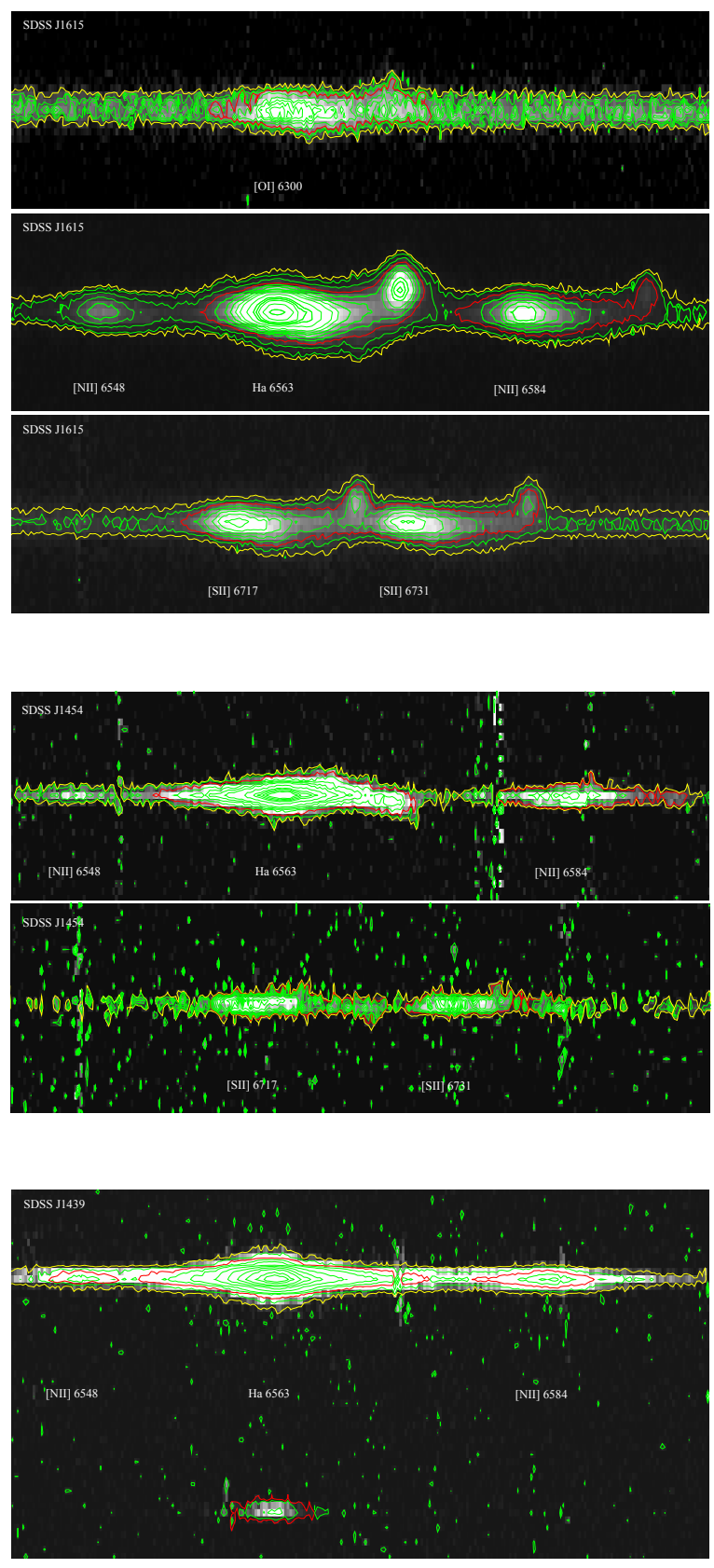

Fig. 1.- 2D-spectra with contours for: J1615 (upper panel) in the region of [O I], $\mathrm{H} \alpha+[\mathrm{N} \mathrm{II}]$, and [S II]; J1454 in the region of $\mathrm{H} \alpha+[\mathrm{N}$ II] and [S II]; and J1439 in the region of $\mathrm{H} \alpha+[\mathrm{N} \mathrm{II}]$. 

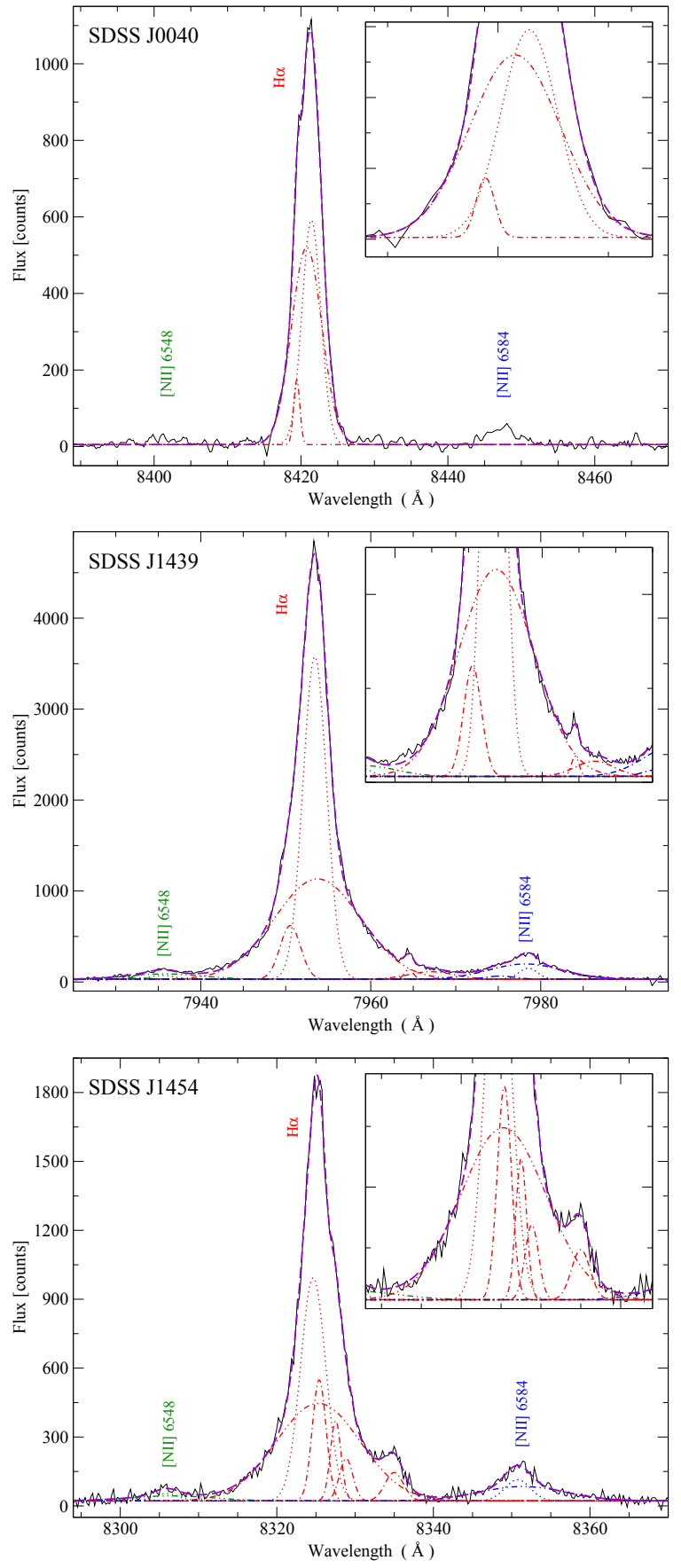

Fig. 2.- Multiple Gaussian fitting in the spectral range around $\mathrm{H} \alpha+[\mathrm{N} \mathrm{II}] \lambda \lambda 6548,6584 \AA$ for the sample galaxies. The inset shows, in each case, a zoom of the bases of the $\mathrm{H} \alpha$ emission line and their NGAUSSFIT components superimposed.
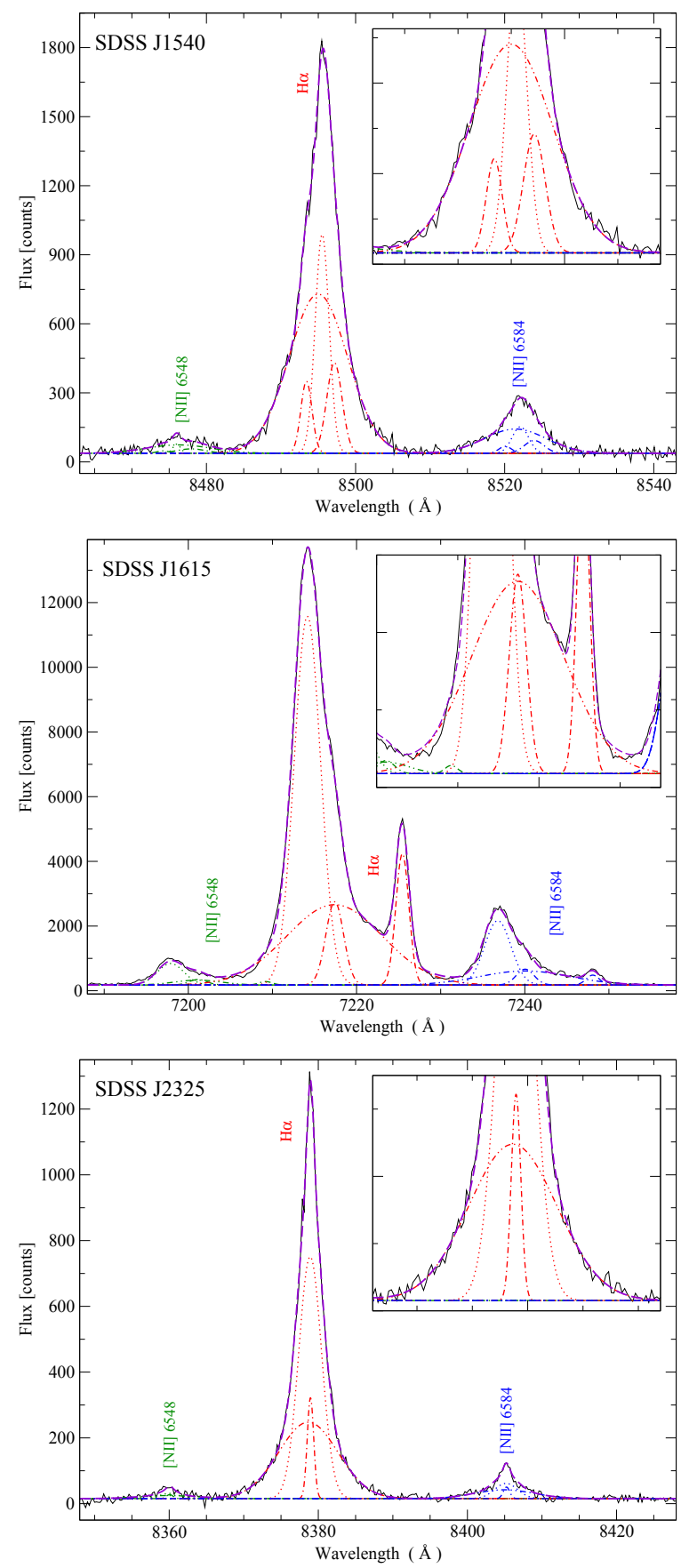

Fig. 3.- Same as Figure 2 

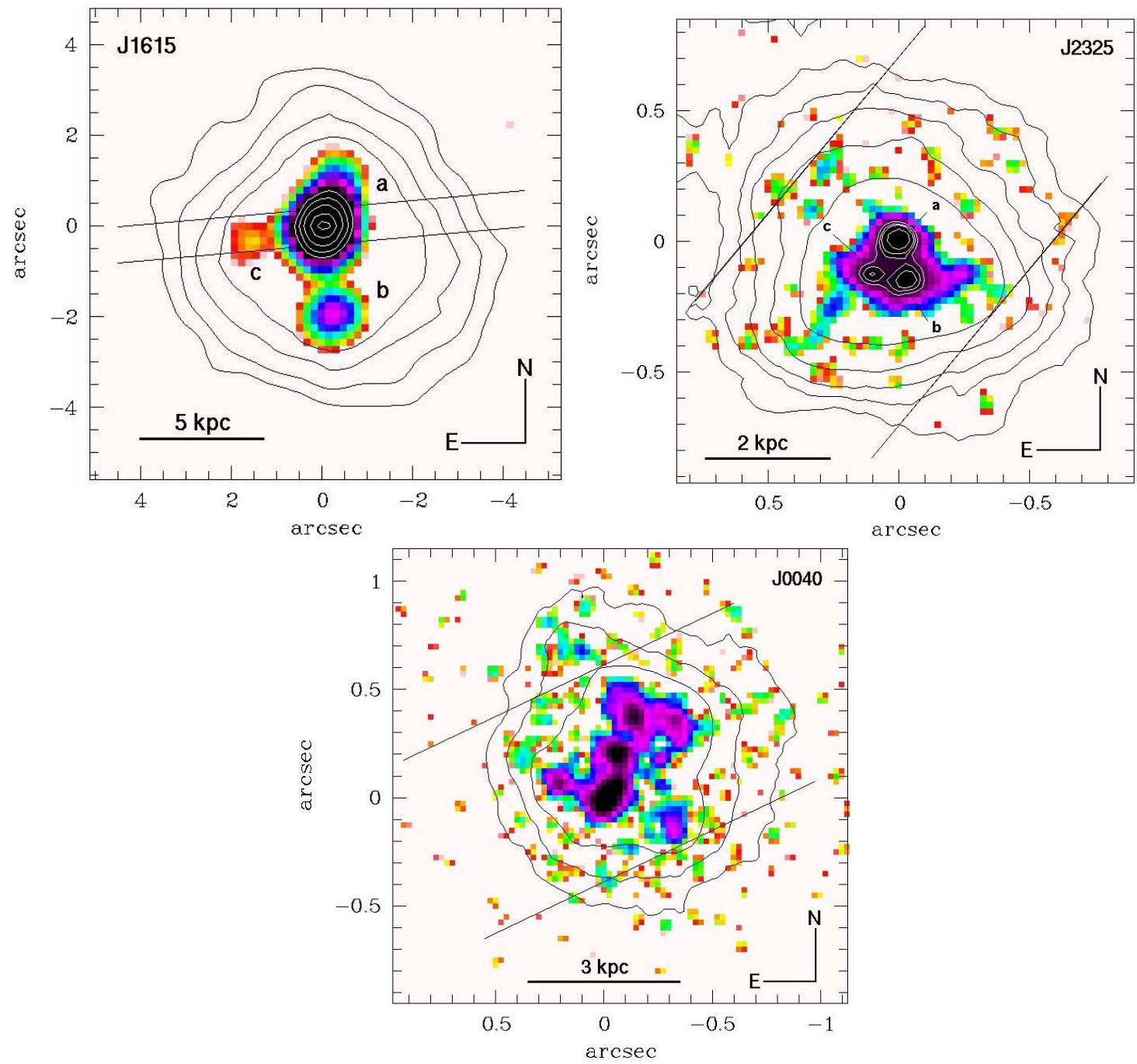

Fig. 4.- SDSS gri (left panel) and HST/ACS F150 (middle and right panels) of three GPs from the present sample after unsharp-masking. The area covered by the longslit aperture is indicated. Contours are computed from the galaxy images prior to unsharp masking and delineate the morphology of the LSB envelope. North is up and east to the left. 
TABLE 1

$\mathrm{H} \alpha$ BROAD AND NARROW COMPONENTS

\begin{tabular}{|c|c|c|c|c|}
\hline $\begin{array}{c}\text { Galaxy }^{\dagger} \\
\text { component }\end{array}$ & $\begin{array}{c}\Delta \mathrm{v}^{\mathrm{a}} \\
\mathrm{km} \mathrm{s}\end{array}$ & $\begin{array}{c}\sigma^{\mathrm{b}} \\
\mathrm{km} \mathrm{s}^{-1}\end{array}$ & $\begin{array}{c}\mathrm{FWZI}^{\mathrm{c}} \\
(\AA) \mathrm{km} \mathrm{s}^{-1}\end{array}$ & $\underset{\%}{\mathrm{EM}_{f}}{ }^{\mathrm{d}}$ \\
\hline \multicolumn{5}{|c|}{ SDSS J004054.31+153409.8 $(=\mathrm{J} 0040), z=0.283$} \\
\hline broad & $-23 \pm 2$ & $94 \pm 2$ & (18) 640 & 56 \\
\hline n1 & $0 \pm 1$ & $57 \pm 1$ & & 40 \\
\hline $\mathrm{n} 2$ & $-71 \pm 2$ & $9 \pm 1$ & & 4 \\
\hline \multicolumn{5}{|c|}{ SDSS J143905.23+245353.3 (=J1439), $z=0.212$} \\
\hline broad & $12 \pm 3$ & $234 \pm 2$ & (39) 1470 & 49 \\
\hline n1 & $0 \pm 1$ & $62 \pm 1$ & & 43 \\
\hline n2 & $-109 \pm 2$ & $53 \pm 2$ & & 6 \\
\hline n3 & $418 \pm 6$ & $16 \pm 5$ & & 1 \\
\hline $\mathrm{n} 4$ & $520 \pm 20$ & $120 \pm 20$ & & 2 \\
\hline \multicolumn{5}{|c|}{ SDSS J145435.57+452856.3 (=J1454), $z=0.268$} \\
\hline broad & $24 \pm 3$ & $248 \pm 2$ & (37) 1330 & 47 \\
\hline n1 & $0 \pm 1$ & $73 \pm 1$ & & 32 \\
\hline $\mathrm{n} 2$ & $28 \pm 2$ & $37 \pm 1$ & & 10 \\
\hline n3 & $104 \pm 2$ & $26 \pm 2$ & & 5 \\
\hline $\mathrm{n} 4$ & $152 \pm 2$ & $35 \pm 2$ & & 3 \\
\hline n5 & $373 \pm 6$ & $51 \pm 3$ & & 3 \\
\hline \multicolumn{5}{|c|}{ SDSS J154050.19+572441.9 (=J1540), $z=0.294$} \\
\hline broad & $-18 \pm 2$ & $180 \pm 2$ & (30) 1060 & 64 \\
\hline n1 & $0 \pm 1$ & $39 \pm 1$ & & 21 \\
\hline $\mathrm{n} 2$ & $58 \pm 2$ & $44 \pm 2$ & & 9 \\
\hline n3 & $-74 \pm 2$ & $30 \pm 2$ & & 6 \\
\hline \multicolumn{5}{|c|}{ SDSS J161555.12+420624.5 (=J1615), $z=0.100$} \\
\hline broad & $134 \pm 4$ & $264 \pm 2$ & (42) 1750 & 38 \\
\hline n1 & $0 \pm 1$ & $71 \pm 1$ & & 47 \\
\hline n2 & $135 \pm 2$ & $46 \pm 2$ & & 7 \\
\hline n3 & $469 \pm 2$ & $32 \pm 2$ & & 8 \\
\hline \multicolumn{5}{|c|}{ SDSS J232539.22+004507.2 (=J2325), $z=0.277$} \\
\hline broad & $-9 \pm 3$ & $183 \pm 3$ & (31) 1110 & 42 \\
\hline n1 & $0 \pm 1$ & $69 \pm 1$ & & 52 \\
\hline $\mathrm{n} 2$ & $2 \pm 1$ & $10 \pm 1$ & & 6 \\
\hline
\end{tabular}

${ }^{\dagger}$ Complete name and redshift from the SDSS.

${ }^{\mathrm{a}} \Delta \mathrm{v}=\mathrm{v}_{\text {obs }}-\mathrm{v}_{\text {comp }}$, where $\mathrm{v}_{\text {obs }}$ and $\mathrm{v}_{\text {comp }}$ are the velocity at the peak of the fitted $\mathrm{H} \alpha$ emission line and the main narrow component, respectively.

${ }^{\mathrm{b}}$ The intrinsic velocity dispersion, $\sigma$, is calculated here as $\sigma=\left(\sigma_{g}^{2}-\sigma_{i}^{2}-\sigma_{t}^{2}\right)^{1 / 2}$, where $\sigma_{g}$ is the velocity dispersion from the fitted Gaussian, and $\sigma_{i}$ and $\sigma_{t}$ are the instrumental and the thermal broadening, respectively.

${ }^{\mathrm{c}} 1 \AA$ at $\lambda_{H \alpha}=6562.8 \AA \sim 45.7(1+z)^{-1} \mathrm{~km} / \mathrm{s}$.

${ }^{\mathrm{d}}$ Fractional emission measures $\left(\mathrm{EM}_{f}\right)$ in per cent. 